Proceedings of the Edinburgh Mathematical Society (2011) 54, 731-747

DOI:10.1017/S0013091509001230

\title{
RETRACTS OF TREES AND FREE LEFT ADEQUATE SEMIGROUPS
}

\author{
MARK KAMBITES \\ School of Mathematics, University of Manchester, Oxford Road, \\ Manchester M13 9PL, UK (mark.kambites@manchester.ac.uk)
}

(Received 8 September 2009)

\begin{abstract}
Recent research of the author has studied edge-labelled directed trees under a natural multiplication operation. The class of all such trees (with a fixed labelling alphabet) has an algebraic interpretation, as a free object in the class of adequate semigroups. We consider here a natural subclass of these trees, defined by placing a restriction on edge orientations, and show that the resulting algebraic structure is a free object in the class of left adequate semigroups. Through this correspondence we establish some structural and algorithmic properties of free left adequate semigroups and monoids, and consequently of the category of all left adequate semigroups.
\end{abstract}

Keywords: directed tree; retract; left adequate semigroup; free algebra; word problem

2010 Mathematics subject classification: Primary 20M10

Secondary 08A10; 08A50

\section{Introduction}

In $[\mathbf{1 0}]$ we introduced and studied an intriguing multiplication operation on a class of edge-labelled, directed combinatorial trees. This multiplication will be described in detail below but, roughly speaking, two trees are combined by identifying a distinguished end vertex on the first tree with a distinguished start vertex on the second and then taking a minimal retract (or core, in the language of graph theory).

We showed in [10] that the set of all such trees (with labels from some fixed alphabet) is a naturally arising algebraic structure: it is a free object (of rank the cardinality of the labelling alphabet) in the class of adequate semigroups. Adequate semigroups, which were introduced by Fountain [5] in the 1970s, are semigroups in which the cancellation properties of elements in general are encapsulated in the cancellation properties of idempotent elements. They form a common generalization of inverse semigroups and cancellative monoids, and their study is a key focus of the York School of semigroup theory. They are usually viewed as algebras of signature $(2,1,1)$, with the binary operation of multiplication augmented with unary operations taking each element to an idempotent sharing its cancellation properties on the left or right respectively.

The author's explicit representation of free adequate semigroups by labelled trees, which may be viewed as analogous to Munn's celebrated representation of the free inverse 
monoid [11], immediately yields detailed structural information about free adequate semigroups, and hence also considerable information about the entire class of adequate semigroups. For example, since multiplication of trees is an effective process, it gives a solution to the word problem for free adequate semigroups, and hence an algorithm to decide whether any given identity is satisfied by every adequate semigroup.

We restrict our attention here to a certain natural subset of labelled trees: those in which every vertex is orientated away from the start vertex. Our main result is that the class of such trees forms a free object in the class of left adequate semigroups (roughly speaking, semigroups in which each element shares its right cancellation properties with an idempotent, usually viewed as $(2,1)$-algebras). As in the two-sided case, this description yields a great deal of information about free left adequate semigroups. An additional corollary is that each free left adequate semigroup embeds into the corresponding free adequate semigroup, from which it follows that every $(2,1)$-identify satisfied by all adequate semigroups is also satisfied by all left adequate semigroups. All of our results about free left adequate semigroups have immediate duals for free right adequate semigroups (which can be represented by those trees in which every vertex is orientated towards the end vertex).

An alternative approach to free left and right adequate semigroups is given by recent work of Branco et al. $[\mathbf{1}, \mathbf{8}]$. Their construction arose from the fact that free left and right adequate semigroups are proper in the sense introduced in $[\mathbf{1}]$.

The remainder of this paper comprises three sections. In $\S 2$ we recall the tree multiplication operations introduced in [10]; we also recall some definitions and elementary properties of adequate semigroups, and the results of [10] characterizing free adequate semigroups as semigroups of trees under multiplication. Section 3 is devoted to the proof that certain subalgebras of the free adequate semigroup are in fact the free left adequate and free right adequate semigroups on the given generating set. Finally, in $\S 4$ we collect together some remarks on and corollaries of our main results.

\section{Preliminaries}

In this section we briefly recall some definitions and key results from [10]; a more detailed exposition may be found in that paper. We also recall the definitions and basic properties of left, right and two-sided adequate semigroups, more details of which can be found in [5].

We are concerned with labelled directed trees, by which we mean edge-labelled directed graphs whose underlying undirected graphs are trees. If $e$ is an edge in such a tree, we denote by $\alpha(e), \omega(e)$ and $\lambda(e)$ the vertex at which $e$ starts, the vertex at which $e$ ends and the label of $e$, respectively.

Let $\Sigma$ be an alphabet. A $\Sigma$-tree (or just a tree if the alphabet $\Sigma$ is clear) is a directed tree with edges labelled by elements of $\Sigma$, and with two distinguished vertices (the start vertex and the end vertex) such that there is a (possibly empty) directed path from the start vertex to the end vertex. Figure 1 shows some examples of $\Sigma$-trees where $\Sigma=\{a, b\}$; in each tree, the start and end vertices are marked by an arrowhead and a cross, respectively. 

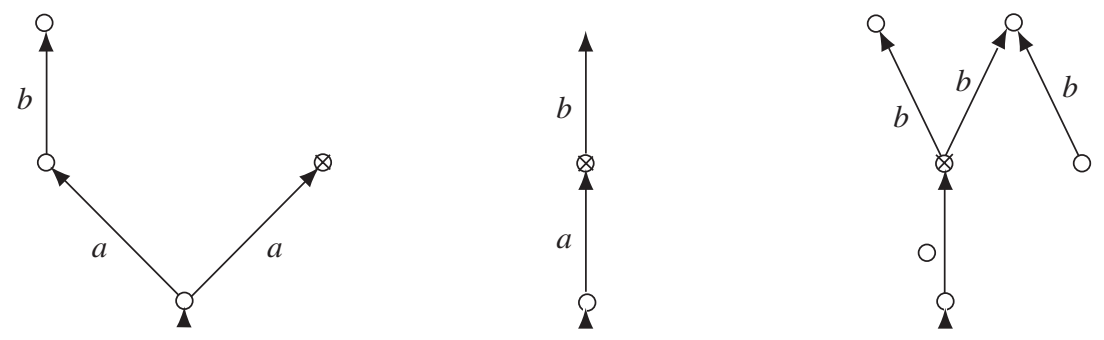

Figure 1. Some examples of $\{a, b\}$-trees.

A tree with only one vertex is called trivial, while a tree with start vertex equal to its end vertex is called idempotent. A tree with a single edge and distinct start and end vertices is called a base tree; we identify each base tree with the label of its edge. In any tree, the (necessarily unique) directed path from the start vertex to the end vertex is called the trunk of the tree; the vertices of the graph which lie on the trunk (including the start and end vertices) are called trunk vertices and the edges which lie on the trunk are called trunk edges. If $X$ is a tree, we write $\theta(X)$ for the set of trunk edges of $X$.

A subtree of a tree $X$ is a subgraph of $X$ containing the start and end vertices, the underlying undirected graph of which is connected. A morphism $\rho: X \rightarrow Y$ of $\Sigma$-trees $X$ and $Y$ is a map taking edges to edges and vertices to vertices, such that $\rho(\alpha(e))=\alpha(\rho(e))$, $\rho(\omega(e))=\omega(\rho(e))$ and $\lambda(e)=\lambda(\rho(e))$ for all edges $e$ in $X$, and which maps the start and end vertices of $X$ to the start and end vertices of $Y$, respectively. Morphisms have the expected properties that the composition of two morphisms (where defined) is again a morphism, while the restriction of a morphism to a subtree is also a morphism. A morphism maps the trunk edges of its domain bijectively onto the trunk edges of its image.

An isomorphism is a morphism which is bijective on both edges and vertices. The set of all isomorphism types of $\Sigma$-trees is denoted $U T^{1}(\Sigma)$, while the set of isomorphism types of non-trivial $\Sigma$-trees is denoted $U T(\Sigma)$. The set of isomorphism types of idempotent trees is denoted $U E^{1}(\Sigma)$, while the set of isomorphism types of non-trivial idempotent trees is denoted $U E(\Sigma)$. Much of the time we shall be formally concerned not with trees themselves but rather with isomorphism types. However, where no confusion is likely, we shall for the sake of conciseness ignore the distinction and implicitly identify trees with their respective isomorphism types.

A retraction of a tree $X$ is an idempotent morphism from $X$ to $X$; its image is a retract of $X$. A tree $X$ is called pruned if it does not admit a non-identity retraction. The set of all isomorphism types of pruned trees (respectively, non-trivial pruned trees) is denoted $T^{1}(\Sigma)$ (respectively, $T(\Sigma)$ ). Just as with morphisms, it is readily verified that the restriction of a retraction to a subtree is again a retraction. Unlike morphisms, a composition of two retractions need not be a retraction, but it will be a retraction, provided the domain of the second is exactly the image of the first. A key foundational result from $[\mathbf{1 0}]$ is the following.

Proposition 2.1 (confluence of retracts). For each tree $X$ there is a unique (up to isomorphism) pruned tree which is a retract of $X$. 


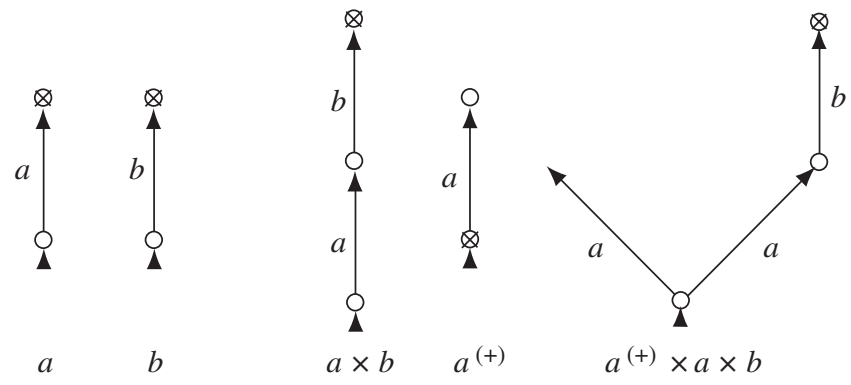

Figure 2. Examples of unpruned operations on $\{a, b\}$-trees.

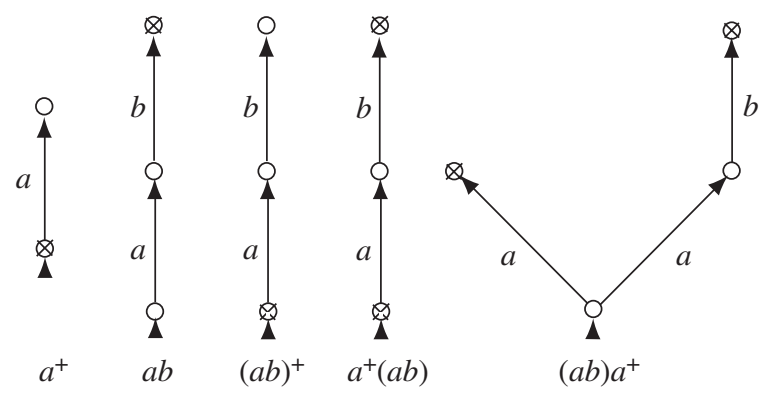

Figure 3. Examples of pruned operations on $\{a, b\}$-trees.

The unique pruned retract of $X$ is called the pruning of $X$ and is denoted $\bar{X}$.

We now define some unpruned operations on (isomorphism types of) trees. If $X, Y \in$ $U T^{1}(\Sigma)$, then $X \times Y$ is (the isomorphism type of) the tree obtained by glueing together $X$ and $Y$, identifying the end vertex of $X$ with the start vertex of $Y$ and keeping all other vertices and all edges distinct. If $X \in U T^{1}(\Sigma)$, then $X^{(+)}$is (the isomorphism type of) the tree with the same labelled graph and start vertex of $X$, but with end vertex the start vertex of $X$. Dually, $X^{(*)}$ is the isomorphism type of the idempotent tree with the same underlying graph and end vertex as $X$, but with start vertex the end vertex of $X$. It was shown in [10] that the unpruned multiplication operation $\times$ is a well-defined associative binary operation on $U T^{1}(\Sigma)$; the (isomorphism type of the) trivial tree is an identity element for this operation, and $U T(\Sigma)$ forms a subsemigroup. The maps $X \mapsto X^{(+)}$and $X \mapsto X^{(*)}$ are well-defined idempotent unary operations on $U T^{1}(\Sigma)$, and the subsemigroup generated by their images is commutative. Figure 2 shows some examples of unpruned operations.

We define corresponding pruned operations on $T^{1}(\Sigma)$ by $X Y=\overline{X \times Y}, X^{*}=\overline{X^{(*)}}$ and $X^{+}=\overline{X^{(+)}}$. These inherit the properties noted above for unpruned operations, and have the additional property that the images of the $*$ and + operations are composed entirely of elements which are idempotent under the multiplication. Figure 3 shows some examples of pruned operations. Notice that the tree for $a^{+}(a b)$ is a proper retract of the tree for $a^{(+)} \times(a \times b)$ from Figure 2. Notice also that the tree for $(a b)^{+} a$ is simply that for $(a \times b)^{(+)} \times a$; it does not admit a non-identity retract.

We recall a key result from $[\mathbf{1 0}]$. 
Theorem 2.2. The pruning map

$$
U T^{1}(\Sigma) \rightarrow T^{1}(\Sigma), \quad X \mapsto \bar{X}
$$

is a surjective $(2,1,1,0)$-morphism from the set of isomorphism types of $\Sigma$-trees under unpruned multiplication, unpruned $(*)$ and unpruned $(+)$ with distinguished identity element to the set of isomorphism types of pruned trees under pruned multiplication, * and + with distinguished identity element.

If $X$ is a tree and $S$ is a set of non-trunk edges and vertices of $X$, then $X \backslash S$ denotes the largest subtree of $X$ (recalling that a subtree must be connected and contain the start and end vertices, and hence the trunk) which does not contain any vertices or edges from $S$. If $s$ is a single edge or vertex we write $X \backslash s$ for $X \backslash\{s\}$. If $u$ and $v$ are vertices of $X$ such that there is a directed path from $u$ to $v$, then we shall denote by $\left.X\right|_{v} ^{u}$ the tree which has the same underlying labelled directed graph as $X$ but start vertex $u$ and end vertex $v$. If $X$ has start vertex $a$ and end vertex $b$, then we define $\left.X\right|^{u}=\left.X\right|_{b} ^{u}$ and $\left.X\right|_{v}=\left.X\right|_{v} ^{a}$ where applicable.

We now recall the definitions of (left, right and two-sided) adequate semigroups. On any semigroup $S$, an equivalence relation $\mathcal{L}^{*}$ is defined by $a \mathcal{L}^{*} b$ if and only if

$$
a x=a y \Longleftrightarrow b x=b y
$$

for every $x, y \in S^{1}$. Dually, an equivalence relation $\mathcal{R}^{*}$ is defined by $a \mathcal{R}^{*} b$ if and only if

$$
x a=y a \Longleftrightarrow x b=y b
$$

for every $x, y \in S^{1}$. A semigroup is called left adequate (right adequate) if every $\mathcal{R}^{*}$-class (respectively, every $\mathcal{L}^{*}$-class) contains an idempotent, and the idempotents commute. A semigroup is adequate if it is both left adequate and right adequate. It is easily seen that in a left (right) adequate semigroup, each $\mathcal{R}^{*}$-class $\left(\mathcal{L}^{*}\right.$-class) must contain a unique idempotent. We denote by $x^{+}$(respectively, $x^{*}$ ) the unique idempotent in the $\mathcal{R}^{*}$-class (respectively, $\mathcal{L}^{*}$-class) of an element $x$; this idempotent acts as a left (right) identity for $x$. The unary operations $x \mapsto x^{+}$and $x \mapsto x^{*}$ are of such critical importance in the theory of adequate (left adequate, right adequate) semigroups that it is usual to consider these semigroups as algebras of signature $(2,1,1)$ (or $(2,1)$ for left adequate and right adequate semigroups) with these operations. In particular, one restricts attention to morphisms that preserve the + and/or $*$ operations (and hence coarsen the $\mathcal{R}^{*}$ and $\mathcal{L}^{*}$ relations) as well as the multiplication. Similarly, adequate (left or right adequate) monoids may be viewed as algebras of signature $(2,1,1,0)[(2,1,0)]$ with the identity a distinguished constant symbol.

The following proposition recalls some basic properties of left and right adequate semigroups; these are well known and full proofs can be found in $[\mathbf{1 0}]$. 
Proposition 2.3. Let $S$ be a left adequate [respectively, right adequate] semigroup and let $a, b, e, f \in S$ with $e$ and $f$ idempotent. Then

(i) $e^{+}=e\left[e=e^{*}\right]$,

(ii) $(a b)^{+}=\left(a b^{+}\right)^{+}\left[(a b)^{*}=\left(a^{*} b\right)^{*}\right]$,

(iii) $a^{+} a=a\left[a a^{*}=a\right]$,

(iv) $e a^{+}=(e a)^{+}\left[a^{*} e=(a e)^{*}\right]$,

(v) $a^{+}(a b)^{+}=(a b)^{+}\left[(a b)^{*} a^{*}=\left(a b^{*}\right)\right]$ and

(vi) if ef $=f$, then $(a e)^{+}(a f)^{+}=(a f)^{+}\left[(e a)^{*}(f a)^{*}=(f a)^{*}\right]$

Recall that an object $F$ in a concrete category $\mathcal{C}$ is called free on a subset $\Sigma \subseteq F$ if every function from $\Sigma$ to an object $N$ in $\mathcal{C}$ extends uniquely to a morphism from $F$ to $N$. The subset $\Sigma$ (which generates $F$ ) is called a free generating set for $F$, and its cardinality is the rank of $F$. The following theorem was the main result of [10].

Theorem 2.4. The monoid $T^{1}(\Sigma)$ is a free adequate monoid, freely generated by the set $\Sigma$ of base trees.

Corollary 2.5. Any subset of $T^{1}(\Sigma)$ closed under the operations of pruned multiplication and + (respectively, $*$ ) forms a left adequate (respectively, right adequate) semigroup under these operations.

It is easily seen that classes of left and right adequate semigroups form a quasivariety, and it follows from general results (see, for example, [4, Proposition VI.4.5]) that free left and right adequate semigroups and monoids exist. Branco et al. $[\mathbf{1 , 8}]$ have recently made the first significant progress in the study of these semigroups. In the present paper we shall give an explicit geometric representation of them. We shall need a proposition, the essence of which is that the distinction between semigroups and monoids is unimportant. The proof is essentially the same as for the corresponding result in the (two-sided) adequate case, which can be found in $[\mathbf{1 0}]$.

Proposition 2.6. Let $\Sigma$ be an alphabet. The free left adequate (free right adequate) monoid on $\Sigma$ is isomorphic to the free left adequate (free right adequate) semigroup on $\Sigma$ with a single adjoined element which is an identity for multiplication and a fixed point for + (respectively, $*$ ).

\section{Free left adequate monoids and semigroups}

In [10] we saw that the monoids $T^{1}(\Sigma)$ and semigroups $T(\Sigma)$ are precisely the free objects in the quasivarieties of adequate monoids and semigroups, respectively. In this section, we prove the main results of the present paper by establishing a corresponding result for left adequate and right adequate monoids and semigroups. The spirit and outline of the proof are similar to those of [10], but the technical details are in places rather different. 
Definition 3.1 (left and right adequate trees). A $\Sigma$-tree $X$ is called left adequate if for each vertex $v$ of $X$ there is a directed path from the start vertex to $v$ or, equivalently, if every non-trunk edge in $X$ is orientated away from the trunk. The sets of isomorphism types of left adequate $\Sigma$-trees and left adequate pruned $\Sigma$-trees are denoted by $L U T^{1}(\Sigma)$ and $L T^{1}(\Sigma)$, respectively.

Dually, a $\Sigma$-tree $X$ is called right adequate if for each vertex $v$ of $X$ there is a directed path from $v$ to the end vertex or, equivalently, if every non-trunk edge in $X$ is orientated towards the trunk. The sets of isomorphism types of right adequate $\Sigma$-trees and right adequate pruned $\Sigma$-trees are denoted $R U T^{1}(\Sigma)$ and $R T^{1}(\Sigma)$, respectively.

Returning to our examples in Figure 1, the left-hand and middle tree are left adequate, while the right-hand tree is not, because of the presence of the rightmost edge which is orientated towards the start vertex. None of the trees shown are right adequate.

From now on we shall work with left adequate trees and left adequate monoids, but of course duals for all of our results apply to right adequate trees and right adequate monoids.

Proposition 3.2. The set $L U T^{1}(\Sigma)$ of left adequate $\Sigma$-trees contains the trivial tree and the base trees, and is closed under unpruned multiplication, unpruned $(+)$ and taking retracts.

Proof. It follows immediately from the definitions that the trivial tree and base trees are left adequate.

Let $X$ and $Y$ be left adequate trees with start vertices $u$ and $v$, respectively. Then $u$ is the start vertex of $X \times Y$, and $X \times Y$ has a directed path from $u$ to $v$. Now, for any vertex $w \in X \times Y$, either $w$ is a vertex of $X$ or $w$ is a vertex of $Y$. In the former case, there is a directed path from $u$ to $w$ in $X$, and hence in $X \times Y$. In the latter case, there is a directed path from $v$ to $w$ in $Y$, and hence in $X \times Y$, which, composed with the path from $u$ to $v$, yields a directed path from $u$ to $w$. Thus, $X \times Y$ is left adequate.

Consider next the tree $X^{(+)}$. This has the same underlying directed graph as $X$ and the same start vertex, so it is immediate that it is left adequate.

Finally, let $\pi: X \rightarrow Y$ be a retraction with image $Y$ a subtree of $X$. Now for any vertex $w$ in $Y$ there is a directed path from the start vertex of $X$ to $w$ in $X$; since $Y$ is a subtree it is connected and has the same start vertex as $X$, so this must also be a path in $Y$. Thus, $Y$ is left adequate.

Proposition 3.3. The set $L T^{1}(\Sigma)$ of pruned left adequate trees is generated as a $(2,1,0)$-algebra (with operations pruned multiplication and pruned + and a distinguished identity element) by the set $\Sigma$ of base trees.

Proof. The proof is similar to the corresponding one in $[\mathbf{1 0}]$, so we describe it only in outline. Let $\langle\Sigma\rangle$ denote the $(2,1,0)$-subalgebra of $L T^{1}(\Sigma)$ generated by $\Sigma$. We show that every left adequate $\Sigma$-tree is contained in $\langle\Sigma\rangle$ by induction on number of edges. The tree with no edges is the identity element of $L T^{1}(\Sigma)$ and so by definition is contained in $\langle\Sigma\rangle$. Now suppose for induction that $X \in L T^{1}(\Sigma)$ has at least one edge, and that every tree in $L T^{1}(\Sigma)$ with strictly fewer edges lies in $\langle\Sigma\rangle$. 
If $X$ has a trunk edge, then let $v_{0}$ be the start vertex of $X, e$ be the trunk edge incident with $v_{0}, a=\lambda(e)$ and $v_{1}=\omega(e)$. Let $Y=\left.X\right|_{v_{0}} ^{v_{0}} \backslash e$ and $Z=\left.X\right|^{v_{1}} \backslash e$. Then $Y$ and $Z$ are pruned trees with strictly fewer edges than $X$, and so by induction lie in $\langle\Sigma\rangle$. Now, clearly, from the definitions we have $Y \times a \times Z=X$, and since $X$ is pruned using Theorem 2.2 we have

$$
Y a Z=\overline{Y \times a \times Z}=\bar{X}=X,
$$

so that $X \in\langle\Sigma\rangle$ as required.

If, on the other hand, $X$ has no trunk edges, then let $e$ be any edge incident with the start vertex $v_{0}$, and suppose $e$ has label $a$. Since the tree is left adequate, $e$ must be orientated away from $v_{0}$; let $v_{1}=\omega(e)$. We define $Y=\left.X\right|_{v_{0}} ^{v_{0}} \backslash e$ and $Z=X||_{v_{1}}^{v_{1}} \backslash e$, and a similar argument to that above shows that $X=Y(a Z)^{+}$, where $Y, Z \in\langle\Sigma\rangle$, so that again $X \in\langle\Sigma\rangle$.

Now suppose $M$ is a left adequate monoid and $\chi: \Sigma \rightarrow M$ is a function. Our objective is to show that there is a unique $(2,1,0)$-morphism from $L T^{1}(\Sigma)$ to $M$ which extends $\chi$. Following the strategy of [10], we begin by defining a map $\tau$ from the set of idempotent left adequate $\Sigma$-trees to the set $E(M)$ of idempotents in the monoid $M$. Let $X$ be an idempotent left adequate $\Sigma$-tree with start vertex $v$. If $X$ has no edges, then we define $\tau(X)=1$. Otherwise, we define $\tau(X)$ recursively, in terms of the value of $\tau$ on left adequate trees with strictly fewer edges than $X$, as follows. Let $E^{+}(X)$ be the set of edges in $X$ which start at the start vertex $v$ and define

$$
\tau(X)=\prod_{e \in E^{+}(X)}\left[\chi(\lambda(e)) \tau\left(\left.X\right|_{\omega(e)} ^{\omega(e)} \backslash e\right)\right]^{+} .
$$

It is easily seen that each $\left.X\right|_{\omega(e)} ^{\omega(e)} \backslash e$ is a left adequate tree with strictly fewer edges than $X$, so this gives a valid recursive definition of $\tau$. Moreover, the product is non-empty and, because idempotents commute in the left adequate monoid $M$, its value is idempotent and independent of the order in which the factors are multiplied. Note that if the left adequate monoid $M$ is in fact adequate, then the function $\tau$ defined here takes the same values on left adequate trees as the corresponding map defined in [10].

Proposition 3.4. Let $X$ be an idempotent left adequate tree with start vertex $v$, and suppose $X_{1}$ and $X_{2}$ are subtrees of $X$ such that $X=X_{1} \cup X_{2}$ and $X_{1} \cap X_{2}=\{v\}$. Then $\tau(X)=\tau\left(X_{1}\right) \tau\left(X_{2}\right)$.

Proof. Clearly, we have $E^{+}(X)=E^{+}\left(X_{1}\right) \cup E^{+}\left(X_{2}\right)$, and for $i \in\{1,2\}$ and $e \in$ $E^{+}\left(X_{i}\right)$ we have

$$
\tau\left(\left.X\right|_{\omega(e)} ^{\omega(e)} \backslash e\right)=\tau\left(\left.X_{i}\right|_{\omega(e)} ^{\omega(e)} \backslash e\right)
$$

so it follows that

$$
\left[\chi(\lambda(e)) \tau\left(\left.X\right|_{\omega(e)} ^{\omega(e)} \backslash e\right)\right]^{+}=\left[\chi(\lambda(e)) \tau\left(\left.X_{i}\right|_{\omega(e)} ^{\omega(e)} \backslash e\right)\right]^{+} .
$$

The claim now follows directly from the definition of $\tau$. 
Corollary 3.5. Let $X$ be an idempotent left adequate tree with start vertex $v$, and let $e$ be an edge incident with $v$. Then

$$
\tau(X)=\tau(X \backslash \omega(e))\left[\chi(\lambda(e)) \tau\left(\left.X\right|_{\omega(e)} ^{\omega(e)} \backslash e\right)\right]^{+} .
$$

Proof. Let $X_{1}=X \backslash e=X \backslash \omega(e)$, let $S$ be the set of edges in $X$ which are incident with $v$ and let $X_{2}=X \backslash(S \backslash\{e\})$ be the maximum subtree of $X$ containing $e$ but none of the other edges incident with $v$. Now, clearly, we have $E^{+}\left(X_{2}\right)=\{e\}$, so by the definition of $\tau$ we have

$$
\tau\left(X_{2}\right)=\left[\chi(\lambda(e)) \tau\left(\left.X\right|_{\omega(e)} ^{\omega(e)} \backslash e\right)\right]^{+} .
$$

We also have $X=X_{1} \cup X_{2}$ and $X_{1} \cap X_{2}=\{v\}$, so, by Proposition 3.4,

$$
\tau(X)=\tau\left(X_{1}\right) \tau\left(X_{2}\right)=\tau(X \backslash \omega(e))\left[\chi(\lambda(e)) \tau\left(\left.X\right|_{\omega(e)} ^{\omega(e)} \backslash e\right)\right]^{+}
$$

as required.

Next we define a map $\rho: L U T^{1}(\Sigma) \rightarrow M$, from the set of isomorphism types of left adequate $\Sigma$-trees to the left adequate monoid $M$. Suppose a tree $X$ has trunk vertices $v_{0}, \ldots, v_{n}$ in sequence. For $1 \leqslant i \leqslant n$ let $a_{i}$ be the label of the edge from $v_{i-1}$ to $v_{i}$. For $0 \leqslant i \leqslant n$ let $X_{i}=\left.X\right|_{v_{i}} ^{v_{i}} \backslash \theta(X)$. We define

$$
\rho(X)=\tau\left(X_{0}\right) \chi\left(a_{1}\right) \tau\left(X_{1}\right) \chi\left(a_{2}\right) \cdots \chi\left(a_{n-1}\right) \tau\left(X_{n-1}\right) \chi\left(a_{n}\right) \tau\left(X_{n}\right) .
$$

Note that in the case $i=0$ (that is, where $X$ is an idempotent tree) we have $\rho(X)=$ $\tau\left(X_{0}\right)=\tau(X)$. Clearly, the value of $\rho$ depends only on the isomorphism type of $X$, so $\rho$ is indeed a well-defined map from $L U T^{1}(\Sigma)$ to $M$. Again, if $M$ is right adequate as well as left adequate, then the function $\rho$ takes the same value on left adequate trees as its counterpart in $[\mathbf{1 0}]$.

Proposition 3.6. Let $X$ be a left adequate tree with trunk vertices $v_{0}, \ldots, v_{n}$ in sequence, where $n \geqslant 1$. Let $a_{1}$ be the label of the edge from $v_{0}$ to $v_{1}$. Then

$$
\rho(X)=\tau\left(\left.X\right|_{v_{0}} ^{v_{0}} \backslash v_{1}\right) \chi\left(a_{1}\right) \rho\left(\left.X\right|^{v_{1}} \backslash v_{0}\right) .
$$

Proof. Let $X_{0}, \ldots, X_{n}$ be as in the definition of $\rho$, so that

$$
\rho(X)=\tau\left(X_{0}\right) \chi\left(a_{1}\right) \tau\left(X_{1}\right) \chi\left(a_{2}\right) \cdots \chi\left(a_{n-1}\right) \tau\left(X_{n-1}\right) \chi\left(a_{n}\right) \tau\left(X_{n}\right) .
$$

It follows straight from the definition that

$$
\rho\left(\left.X\right|^{v_{1}} \backslash v_{0}\right)=\tau\left(X_{1}\right) \chi\left(a_{2}\right) \cdots \chi\left(a_{n-1}\right) \tau\left(X_{n-1}\right) \chi\left(a_{n}\right) \tau\left(X_{n}\right),
$$

so we have

$$
\begin{aligned}
\rho(X) & =\tau\left(X_{0}\right) \chi\left(a_{1}\right) \rho\left(\left.X\right|^{v_{1}} \backslash v_{0}\right) \\
& =\tau\left(\left.X\right|_{v_{0}} ^{v_{0}} \backslash v_{1}\right) \chi\left(a_{1}\right) \rho\left(\left.X\right|^{v_{1}} \backslash v_{0}\right)
\end{aligned}
$$

as required. 
Proposition 3.7. The map $\rho: L U T^{1}(\Sigma) \rightarrow M$ is a morphism of $(2,1,0)$-algebras.

Proof. Let $X$ and $Y$ be trees with, say, trunk vertices $u_{0}, \ldots, u_{m}$ and $v_{0}, \ldots, v_{n}$ in sequence, respectively. For each $1 \leqslant i \leqslant m$ let $a_{i}$ be the label of the edge from $u_{i-1}$ to $u_{i}$, and for each $1 \leqslant i \leqslant n$ let $b_{i}$ be the label of the edge from $v_{i-1}$ to $v_{i}$. For each $0 \leqslant i \leqslant m$ let $X_{i}=\left.X\right|_{u_{i}} ^{u_{i}} \backslash \theta(X)$, and, similarly, for each $0 \leqslant i \leqslant n$ define $Y_{i}=\left.Y\right|_{v_{i}} ^{v_{i}} \backslash \theta(Y)$.

Consider now the unpruned product $X \times Y$. It is easily seen that for $0 \leqslant i<m$ we have

$$
\left.(X \times Y)\right|_{u_{i}} ^{u_{i}} \backslash \theta(X \times Y)=X_{i},
$$

while for $0<i \leqslant n$ we have

$$
\left.(X \times Y)\right|_{v_{i}} ^{v_{i}} \backslash \theta(X \times Y)=Y_{i} .
$$

Considering now the remaining trunk vertex $u_{m}=v_{0}$ of $X \times Y$, we have

$$
\left.(X \times Y)\right|_{u_{m}} ^{u_{m}} \backslash \theta(X \times Y)=\left.(X \times Y)\right|_{v_{0}} ^{v_{0}} \backslash \theta(X \times Y)=X_{m} \times Y_{0} .
$$

By Proposition 3.4 and the definition of unpruned multiplication we have $\tau\left(X_{m} \times Y_{0}\right)=$ $\tau\left(X_{m}\right) \tau\left(Y_{0}\right)$. So using the definition of $\rho$ we have

$$
\begin{aligned}
\rho(X \times Y) & =\tau\left(X_{0}\right) \chi\left(a_{1}\right) \tau\left(X_{1}\right) \cdots \chi\left(a_{m}\right) \tau\left(X_{m} \times Y_{0}\right) \chi\left(b_{1}\right) \tau\left(Y_{1}\right) \chi\left(b_{2}\right) \cdots \chi\left(b_{n}\right) \tau\left(Y_{n}\right) \\
& =\tau\left(X_{0}\right) \chi\left(a_{1}\right) \tau\left(X_{1}\right) \cdots \chi\left(a_{m}\right) \tau\left(X_{m}\right) \tau\left(Y_{0}\right) \chi\left(b_{1}\right) \tau\left(Y_{1}\right) \chi\left(b_{2}\right) \cdots \chi\left(b_{n}\right) \tau\left(Y_{n}\right) \\
& =\rho(X) \rho(Y) .
\end{aligned}
$$

Next we claim that $\rho\left(X^{(+)}\right)=\rho(X)^{+}$. We prove this by induction on the number of trunk edges in $X$. If $X$ has no trunk edges, then $X=X^{(+)}$and so, using the fact that $\tau(X) \in E(M)$ is fixed by the + operation in $M$, we have

$$
\rho\left(X^{(+)}\right)=\rho(X)=\tau(X)=\tau(X)^{+}=\rho(X)^{+} .
$$

Now suppose for induction that $X$ has at least one trunk edge and that the claim holds for trees with strictly fewer trunk edges. Recall that

$$
X_{0}=\left.X\right|_{u_{0}} ^{u_{0}} \backslash \theta(X)=\left.X\right|_{u_{0}} ^{u_{0}} \backslash u_{1}
$$

and let $Z=\left.X\right|^{u_{1}} \backslash u_{0}$. Now

$$
\begin{aligned}
\rho\left(X^{(+)}\right) & =\tau\left(X^{(+)}\right) & & \text {(by the definition of } \rho) \\
& =\tau\left(X_{0}\right)\left[\chi\left(a_{1}\right) \tau\left(Z^{(+)}\right)\right]^{+} & & \text {(by Corollary 3.5) } \\
& =\tau\left(X_{0}\right)\left[\chi\left(a_{1}\right) \rho\left(Z^{(+)}\right)\right]^{+} & & \text {(by the definition of } \rho) \\
& =\tau\left(X_{0}\right)\left[\chi\left(a_{1}\right) \rho(Z)^{+}\right]^{+} & & \text {(by the inductive hypothesis) } \\
& =\tau\left(X_{0}\right)\left[\chi\left(a_{1}\right) \rho(Z)\right]^{+} & & \text {(by Proposition 2.3(ii)) } \\
& =\left[\tau\left(X_{0}\right) \chi\left(a_{1}\right) \rho(Z)\right]^{+} & & \text {(by Proposition 2.3 (iv)) } \\
& =\rho(X)^{+} & & \text {(by Proposition 3.6) }
\end{aligned}
$$

as required. 
Finally, it follows directly from the definition that $\rho$ maps the identity element in $L U T^{1}(\Sigma)$ (that is, the isomorphism type of the trivial tree) to the identity of $M$, and so is a $(2,1,0)$-morphism.

So far, we have closely followed the proof strategy from [10], but at this point it becomes necessary to diverge. This is because the arguments employed in the two-sided case involve operations on trees that do not preserve left adequacy, and hence use the * operation in the monoid $M$ even when starting with left adequate trees. Instead, the following lemma about left adequate trees (which fails for general trees) allows us to follow an alternative inductive strategy.

Lemma 3.8. Let $\mu: X \rightarrow Y$ be a morphism of left adequate trees, let $e$ be an edge in $X$ and let $v$ be a vertex of $X$ such that there is a directed path from $\omega(e)$ to $v$. Then

$$
\left.\mu\left(\left.X\right|_{v} ^{\omega(e)} \backslash e\right) \subseteq Y\right|_{\mu(v)} ^{\mu(\omega(e))} \backslash \mu(e)
$$

Proof. Let $X^{\prime}=\left.X\right|_{v} ^{\omega(e)} \backslash e$ and $Y^{\prime}=\left.Y\right|_{\mu(v)} ^{\mu(\omega(e))} \backslash \mu(e)$. Notice first that the image $\mu\left(X^{\prime}\right)$ is connected and contains $\mu(\omega(e))$. Since the underlying undirected graph of $Y$ is a tree, this means that $\mu\left(X^{\prime}\right)$ is either contained in $Y^{\prime}$ as required, or contains the edge $\mu(e)$; suppose for a contradiction that the latter holds, say $\mu(e)=\mu(f)$ for some edge $f$ in $X^{\prime}$. Now, since $X$ is left adequate, there must be a directed path from the start vertex to $\alpha(f)$. But again $e$ is orientated away from the start vertex, and $\alpha(f)$ is in $X^{\prime}$, which is a connected component of $X$ including $\omega(e)$ but not $e$, so this path must clearly pass through the edge $e$. Let $P$ denote the suffix of this path which leads from $\omega(e)$ to $\alpha(f)$. Then $\mu(e P)$ is a non-empty directed path in $Y$ from $\mu(\alpha(e))$ to $\mu(\alpha(f))=\mu(\alpha(e))$, which contradicts the fact that $Y$ is a directed tree.

Lemma 3.9. Suppose that $\mu: X \rightarrow Y$ is a morphism of idempotent left adequate trees. Then $\tau(Y) \tau(X)=\tau(Y)$.

Proof. We use induction on the number of edges in $X$. If $X$ has no edges, then we have $\tau(X)=1$ so the result is clear. Now suppose $X$ has at least one edge and for induction that the result holds for trees $X$ with strictly fewer edges. By the definition of $\tau$ we have

$$
\tau(X)=\prod_{e \in E^{+}(X)}\left[\chi(\lambda(e)) \tau\left(\left.X\right|_{\omega(e)} ^{\omega(e)} \backslash e\right)\right]^{+}
$$

while

$$
\tau(Y)=\prod_{f \in E^{+}(Y)}\left[\chi(\lambda(f)) \tau\left(\left.Y\right|_{\omega(f)} ^{\omega(f)} \backslash f\right)\right]^{+}
$$

Suppose now that $e \in E^{+}(X)$. Then, since $\mu$ is a morphism, the edge $\mu(e)$ lies in $E^{+}(Y)$, and the above expression from $\tau(Y)$ contains a factor

$$
\left[\chi(\lambda(\mu(e))) \tau\left(\left.Y\right|_{\omega(\mu(e))} ^{\omega(\mu(e))} \backslash \mu(e)\right)\right]^{+}
$$


We claim that the factor corresponding to $e$ in the above expression for $\tau(X)$ is absorbed into this factor.

Let $X^{\prime}=\left.X\right|_{\omega(e)} ^{\omega(e)} \backslash e$ and $Y^{\prime}=\left.Y\right|_{\omega(\mu(e))} ^{\omega(\mu(e))} \backslash \mu(e)$. By Lemma 3.8, the morphism $\mu$ restricts to a morphism $\mu^{\prime}: X^{\prime} \rightarrow Y^{\prime}$. Since $X^{\prime}$ has strictly fewer edges than $X$, the inductive hypothesis tells us that $\tau\left(X^{\prime}\right) \tau\left(Y^{\prime}\right)=\tau\left(Y^{\prime}\right)$. Now, by Proposition 2.3 (vi), we have

$$
\left[\chi(\lambda(e)) \tau\left(X^{\prime}\right)\right]^{+}\left[\chi(\lambda(e)) \tau\left(Y^{\prime}\right)\right]^{+}=\left[\chi(\lambda(e)) \tau\left(Y^{\prime}\right)\right]^{+} .
$$

as required.

Corollary 3.10. Let $X$ be a subtree of an idempotent left adequate tree $Y$. Then $\tau(Y) \tau(X)=\tau(Y)$.

Proof. The embedding of $X$ into $Y$ satisfies the conditions of Lemma 3.9.

Corollary 3.11. Let $Y$ be a retract of an idempotent left adequate tree $X$. Then $\tau(X)=\tau(Y)$.

Proof. Let $\pi: X \rightarrow X$ be a retract with image $Y$. Since $\pi$ is a morphism, Lemma 3.9 tells us that $\tau(X) \tau(\pi(X))=\tau(\pi(X))=\tau(Y)$. But, since $\pi(X)$ is a subgraph of $X$, Corollary 3.10 yields $\tau(X) \tau(\pi(X))=\tau(X)$.

Lemma 3.12. Let $X$ be a left adequate tree with trunk vertices $v_{0}, \ldots, v_{n}$ in sequence, where $n \geqslant 0$. Then

$$
\rho(X)=\tau\left(\left.X\right|_{v_{0}} ^{v_{0}}\right) \rho(X) .
$$

Proof. We use induction on the number of trunk edges in $X$. Let $X^{\prime}=\left.X\right|_{v_{0}} ^{v_{0}}$. Clearly, if $X$ has no trunk edges, then we have $X=X^{\prime}$ and from the definition of $\rho$ we have $\rho(X)=\tau\left(X^{\prime}\right)$, so the claim reduces to the fact that $\tau\left(X^{\prime}\right)$ is idempotent. Now suppose that $X$ has at least one trunk edge and that the claim holds for $X$ with strictly fewer trunk edges. Let $Y=\left.X\right|^{v_{1}} \backslash v_{0}$, let $Y^{\prime}=\left.Y\right|_{v_{1}} ^{v_{1}}$ and let $X_{0}=\left.X\right|_{v_{0}} ^{v_{0}} \backslash v_{1}$. Let $a_{1}$ be the label of the edge from $v_{0}$ to $v_{1}$. By Corollary 3.5 we have

$$
\tau\left(X^{\prime}\right)=\left[\chi\left(a_{1}\right) \tau\left(Y^{\prime}\right)\right]^{+} \tau\left(X_{0}\right) .
$$

Now by Proposition 3.6 we deduce that $\rho(X)=\tau\left(X_{0}\right) \chi\left(a_{1}\right) \rho(Y)$. Also, by the inductive hypothesis, we have $\rho(Y)=\tau\left(Y^{\prime}\right) \rho(Y)$. Putting these observations together we have

$$
\begin{aligned}
\tau\left(X^{\prime}\right) \rho(X) & =\left(\left[\chi\left(a_{1}\right) \tau\left(Y^{\prime}\right)\right]^{+} \tau\left(X_{0}\right)\right)\left(\tau\left(X_{0}\right) \chi\left(a_{1}\right) \rho(Y)\right) \\
& =\left[\chi\left(a_{1}\right) \tau\left(Y^{\prime}\right)\right]^{+} \tau\left(X_{0}\right) \chi\left(a_{1}\right)\left[\tau\left(Y^{\prime}\right) \rho(Y)\right] \\
& =\tau\left(X_{0}\right)\left[\chi\left(a_{1}\right) \tau\left(Y^{\prime}\right)\right]^{+}\left[\chi\left(a_{1}\right) \tau\left(Y^{\prime}\right)\right] \rho(Y) \\
& =\tau\left(X_{0}\right) \chi\left(a_{1}\right) \tau\left(Y^{\prime}\right) \rho(Y) \\
& =\tau\left(X_{0}\right) \chi\left(a_{1}\right) \rho(Y) \\
& =\rho(X)
\end{aligned}
$$

as required. 
Corollary 3.13. Let $X$ be a left adequate tree with trunk vertices $v_{0}, \ldots, v_{n}$ in sequence, where $n \geqslant 1$. Let $a_{1}$ be the label of the edge from $v_{0}$ to $v_{1}$. Then

$$
\rho(X)=\tau\left(\left.X\right|_{v_{0}} ^{v_{0}}\right) \chi\left(a_{1}\right) \rho\left(\left.X\right|^{v_{1}} \backslash v_{0}\right) .
$$

Proof. We have

$$
\begin{aligned}
\rho(X) & =\tau\left(\left.X\right|_{v_{0}} ^{v_{0}}\right) \rho(X) & & \text { (by Lemma 3.12) } \\
& =\tau\left(\left.X\right|_{v_{0}} ^{v_{0}}\right) \tau\left(\left.X\right|_{v_{0}} ^{v_{0}} \backslash v_{1}\right) \chi\left(a_{1}\right) \rho\left(\left.X\right|^{v_{1}} \backslash v_{0}\right) & & \text { (by Proposition 3.6) } \\
& =\tau\left(\left.X\right|_{v_{0}} ^{v_{0}}\right) \chi\left(a_{1}\right) \rho\left(\left.X\right|^{v_{1}} \backslash v_{0}\right) & & \text { (by Corollary 3.10). }
\end{aligned}
$$

Proposition 3.14. Let $X$ be a left adequate tree. Then $\rho(X)=\rho(\bar{X})$.

Proof. Let $\pi: X \rightarrow X$ be a retraction with image $\bar{X}$. Suppose $X$ has trunk vertices $v_{0}, \ldots, v_{n}$. For $1 \leqslant i \leqslant n$ let $a_{i}$ be the label of the edge from $v_{i-1}$ to $v_{i}$. We prove the claim by induction on the number of trunk edges in $X$. If $X$ has no trunk edges, then by the definition of $\rho$ and Corollary 3.11 we have

$$
\rho(X)=\tau(X)=\tau(\pi(X))=\rho(\pi(X)) .
$$

Next suppose that $X$ has at least one trunk edge, that is, that $n \geqslant 1$. Let $Z=\left.X\right|^{v_{1}} \backslash v_{0}$. Then by Lemma 3.8 we have

$$
\pi(Z)=\left.\pi\left(\left.X\right|^{v_{1}} \backslash v_{0}\right) \subseteq \pi(X)\right|^{v_{1}} \backslash v_{0}=\left.\bar{X}\right|^{v_{1}} \backslash v_{0}
$$

and, since $\pi$ is idempotent with image $\bar{X}$, the converse inclusion also holds and we have

$$
\pi(Z)=\left.\bar{X}\right|^{v_{1}} \backslash v_{0} .
$$

Moreover, by Lemma 3.8 again, the retraction $\pi$ restricts to a morphism $\pi^{\prime}: Z \rightarrow Z$. Clearly, this morphism must also be a retraction, and $Z$ has strictly fewer edges than $X$, so by the inductive hypothesis and Proposition 2.1 we have

$$
\rho(Z)=\rho(\bar{Z})=\rho\left(\overline{\pi^{\prime}(Z)}\right)=\rho\left(\pi^{\prime}(Z)\right)=\rho(\pi(Z)) .
$$

It also follows easily from definitions that

$$
\pi\left(\left.X\right|_{v_{0}} ^{v_{0}}\right)=\left.\bar{X}\right|_{v_{0}} ^{v_{0}} .
$$

Now

$$
\begin{aligned}
\rho(X) & =\tau\left(\left.X\right|_{v_{0}} ^{v_{0}}\right) \chi\left(a_{1}\right) \rho(Z) & & \text { (by Corollary 3.13) } \\
& =\tau\left(\left.X\right|_{v_{0}} ^{v_{0}}\right) \chi\left(a_{1}\right) \rho(\pi(Z)) & & \text { (by }(3.2)) \\
& =\tau\left(\pi\left(\left.X\right|_{v_{0}} ^{v_{0}}\right)\right) \chi\left(a_{1}\right) \rho(\pi(Z)) & & \text { (by Corollary 3.11) } \\
& =\tau\left(\left.\bar{X}\right|_{v_{0}} ^{v_{0}}\right) \chi\left(a_{1}\right) \rho\left(\left.\bar{X}\right|^{v_{1}} \backslash v_{0}\right) & & \text { (by (3.1) and }(3.3)) \\
& =\rho(\bar{X}) & & \text { (by Corollary 3.13). }
\end{aligned}
$$


Now let $\hat{\rho}: L T^{1}(\Sigma) \rightarrow M$ be the restriction of $\rho$ to the set of (isomorphism types of) pruned left adequate trees.

Corollary 3.15. The function $\hat{\rho}$ is a $(2,1,0)$-morphism from $L T^{1}(\Sigma)$ (with pruned operations) to the left adequate monoid $M$.

Proof. For any $X, Y \in L T^{1}(\Sigma)$, by Theorem 2.2 and Propositions 3.7 and 3.14 we have

$$
\hat{\rho}(X Y)=\rho(X Y)=\rho(\overline{X \times Y})=\rho(X \times Y)=\rho(X) \rho(Y)=\hat{\rho}(X) \hat{\rho}(Y)
$$

and, similarly,

$$
\hat{\rho}\left(X^{+}\right)=\rho\left(\overline{X^{(+)}}\right)=\rho\left(X^{(+)}\right)=\rho(X)^{+}=\hat{\rho}(X)^{+} .
$$

Finally, that $\hat{\rho}$ maps the identity of $L T^{1}(\Sigma)$ to the identity of $M$ is immediate from the definitions.

We are now ready to prove the main results of this paper, which give a concrete description of the free left adequate monoid and free right adequate monoid on a given generating set.

Theorem 3.16. Let $\Sigma$ be a set. Then $L T^{1}(\Sigma)\left(R T^{1}(\Sigma)\right)$ is a free object in the quasivariety of left (right) adequate monoids, freely generated by the set $\Sigma$ of base trees.

Proof. We prove the claim in the left adequate case, the right adequate case being dual. By Corollary 2.5, $L T^{1}(\Sigma)$ is a left adequate monoid. Now for any left adequate monoid $M$ and function $\chi: \Sigma \rightarrow M$, define $\hat{\rho}: L T^{1}(\Sigma) \rightarrow M$ as above. By Corollary 3.15, $\hat{\rho}$ is a $(2,1,0)$-morphism, and it is immediate from the definitions that $\hat{\rho}(a)=\chi(a)$ for every $a \in \Sigma$, so that $\hat{\rho}$ extends $\chi$. Finally, by Proposition $3.3, \Sigma$ is a $(2,1,0)$-algebra generating set for $L T^{1}(\Sigma)$; it follows that the morphism $\hat{\rho}$ is uniquely determined by its restriction to the set $\Sigma$ of base trees, and hence is the unique morphism with the claimed properties.

Combining this result with Proposition 2.6, we also immediately obtain a description of the free left adequate and free right adequate semigroups.

Theorem 3.17. Let $\Sigma$ be a set. Then the $L T(\Sigma)(R T(\Sigma))$ is a free object in the quasivariety of left (right) adequate semigroups, freely generated by the set $\Sigma$ of base trees.

We also have the following relationship between free adequate, free left adequate and free right adequate semigroups and monoids.

Theorem 3.18. Let $\Sigma$ be a set. The free left adequate semigroup (monoid) on $\Sigma$ and free right adequate semigroup (monoid) on $\Sigma$ embed into the free adequate semigroup (monoid) on $\Sigma$ as the (2,1)-subalgebras ((2,1,0)-subalgebras) generated by the free generators under the appropriate operations. Their intersection is the free semigroup (monoid) on $\Sigma$. 


\section{Remarks and consequences}

In this section we collect together some remarks on and consequences of the results in $\S 3$ and their proofs.

In a left adequate tree, the requirement that there be a path from the start vertex to every other vertex uniquely determines the orientation on every edge in the tree. Conversely, every edge-labelled undirected tree with given start and end vertices admits an orientation on the edges which makes it left adequate. It might superficially seem attractive, then, to identify elements of $L U T^{1}(\Sigma)$ with undirected edge-labelled trees with distinguished start and end vertices. However, the reader may easily convince themselves that not every retraction of such a tree defines a retraction of the corresponding directed tree. So, in order to define pruning and multiplication it would be necessary to reinstate the orientation on the edges, which negates any advantage in dropping the orientation in the first place.

The construction in $\S 3$ of a morphism from $L T^{1}(\Sigma)$ to a monoid $M$ depends only on the facts that $M$ is associative with commuting idempotents, and that the + operation is idempotent with idempotent and commutative image and satisfies the six properties given in the case of left adequate semigroups by Proposition 2.3. So a free left adequate semigroup is also free in any class of $(2,1,0)$-algebras that contains it and satisfies these conditions. This includes in particular the class of left Ehresmann semigroups.

As observed in [10], the classes of monoids we have studied can be generalized to give corresponding classes of small categories. A natural extension of our methods can be used to describe the free left adequate and free right adequate category generated by a given directed graph. Just as in the previous remark, the free left adequate category will also be the free left Ehresmann category. Left Ehresmann categories are generalizations of the restriction categories studied by Cockett and Lack [3], which in the terminology of semigroup theory are weakly left E-ample categories [9]. The generalization of our results to categories thus relates to our main results in the same way that the description of the free restriction category on a graph given in $[\mathbf{2}]$ relates to the descriptions of free left ample monoids given by Fountain et al. $[\mathbf{6}, \mathbf{7}]$.

To conclude, we note some properties of free left and right adequate semigroups and monoids, which are obtained by combining Theorem 3.18 with results about free adequate semigroups and monoids that were obtained in [10]. First of all, we saw in [10] that every finitely generated free adequate semigroup has decidable word problem, so Theorem 3.18 implies the following.

Theorem 4.1. The word problem for any finitely generated free left or right adequate semigroup or monoid is decidable.

As in the two-sided case, the exact computational complexity of this problem remains unclear and deserves further study.

Notice that there is a natural correspondence between equations that hold in a free algebra and identities (in the sense of universal algebra) that hold in the corresponding category of algebras. Indeed, any identity which holds in all algebras in a category must in particular hold in its free algebras, so replacing the variables with generators for a 
free algebra of the appropriate rank yields an equation that holds in the free algebra. Conversely, if an equation holds in a free algebra, then we may obtain from it an identity by replacing each generator with a variable; if this identity fails to hold in some algebra $A$, then we have a map from the generators of the free algebra to $A$ that does not extend to a morphism, from which we conclude that $A$ cannot lie in the appropriate category. Combining this with Theorem 4.1 yields the following.

Corollary 4.2. There is an algorithm to decide whether a given $(2,1,0)$-identity holds in every left adequate monoid.

The same reasoning applied to Theorem 3.18 gives the following consequence.

Theorem 4.3. Any $(2,1,0)$-identity which holds in every adequate monoid also holds in every left adequate monoid.

Recall that an equivalence relation $\mathcal{J}$ is defined on any semigroup by $a \mathcal{J} b$ if and only if $a$ and $b$ generate the same principal two-sided ideal. A semigroup is called $\mathcal{J}$-trivial if no two elements generate the same principal two-sided ideal.

Theorem 4.4. Every free left adequate or free right adequate semigroup or monoid is $\mathcal{J}$-trivial.

Proof. If distinct left (right) adequate $\Sigma$-trees $X$ and $Y$ are $\mathcal{J}$-related in $L T^{1}(\Sigma)$ $\left(R T^{1}(\Sigma)\right)$, then they are $\mathcal{J}$-related in the free adequate monoid $T^{1}(\Sigma)$; but we saw in $[\mathbf{1 0}]$ that $T^{1}(\Sigma)$ is $\mathcal{J}$-trivial.

Theorem 4.5. No free left adequate or free right adequate semigroup or monoid on a non-empty set is finitely generated as a semigroup or monoid.

Proof. We saw in $[\mathbf{1 0}]$ that finite subsets of $T^{1}(\Sigma)$ generate subsemigroups whose trees have a bound on the maximum distance of any vertex from the trunk. Since $L T^{1}(\Sigma)$ and $R T^{1}(\Sigma)$ are subsemigroups containing trees with vertices arbitrarily far from the trunk, it follows that they cannot even be contained in finitely generated subsemigroups of $T^{1}(\Sigma)$, let alone be finitely generated themselves.

Acknowledgements. This research was supported by an RCUK Academic Fellowship. The author thanks John Fountain and Victoria Gould for their encouragement and advice, all the authors of [1] for allowing him access to their unpublished work and work in progress, Mark Lawson for alerting him to the existence of work on free restriction categories [2], and the anonymous referee for careful reading of the first draft.

\section{References}

1. M. J. J. Branco, G. M. S. Gomes And V. A. R. Gould, Structure of left adequate and left Ehresmann monoids, Int. J. Alg. Comput. (in press).

2. J. R. B. Cockett And X. Guo, Stable meet semilattice fibrations and free restriction categories, Theory Applic. Categories 16(15) (2006), 307-341.

3. J. R. B. Cockett And S. LACK, Restriction categories, I, Categories of partial maps, Theor. Comp. Sci. 270 (2002), 223-259. 
4. P. M. Cohn, Universal algebra, 2nd edn, Mathematics and Its Applications, Volume 6 (D. Reidel, Dordrecht, 1981).

5. J. B. Fountain, Adequate semigroups, Proc. Edinb. Math. Soc. 22 (1979), 113-125.

6. J. B. Fountain, Free right type A semigroups, Glasgow Math. J. 33 (1991), 135-148.

7. J. B. Fountain, G. M. S. Gomes and V. A. R. Gould, The free ample monoid, Int. J. Alg. Comput. 19(4) (2009), 527-554.

8. G. M. S. Gomes And V. A. R. Gould, Left adequate and left Ehresmann monoids, II, preprint (available at www-users.york.ac.uk/ varg1/properadequatefinal.pdf).

9. V. A. R. Gould, (Weakly) left E-ample semigroups, preprint (available at wwwusers.york.ac.uk/ varg1/finitela.ps).

10. M. Kambites, Free adequate semigroups, J. Austral. Math. Soc. (in press).

11. W. D. Munn, Free inverse semigroups, Proc. Lond. Math. Soc. 29 (1974), 385-404. 
